\title{
Utilising User-Centred Design to Develop a Clinical Guidelines Mobile Application
}

\author{
James Mitchell \\ School of Computing and Mathematics \\ Keele University, \\ Keele, UK \\ j.a.mitchell@keele.ac.uk
}

\begin{abstract}
Clinical guidelines are utilised by clinicians worldwide. Despite widespread use, accessing clinical guidance and information can be highly inefficient and restrictive. At present, there are no 'standards' (clear methods, designs, recommendations) relating to clinical guidelines for use on mobile devices. Previous studies have investigated the delivery of clinical guidelines on mobile devices, but rarely implement well-known heuristics for design and often fail to involve users in each aspect of design and development, leading to poor information usability. To investigate solutions, a user-centred design approach was utilised to inform the design of a smartphone application to deliver clinical guidelines.
\end{abstract}

User-Centred Design, $\mathrm{HCl}$, Mobile Application Development, Clinical Information Systems, Clinical Guidelines.

\section{INTRODUCTION}

\subsection{Problem Statement}

At present, Bedside Clinical Guidelines (BCGs) are available within NHS Trusts across the UK (Pantin et al. 2006). They are designed for "in the moment" use: the user must navigate rapidly to the information required; read; accurately assimilate the information; and finally, take appropriate action. The guidelines are currently produced as an A5 book, which is usually located at nursing stations or in a Doctor's possession. They are also available as an eBook via the NHS Trust Intranet. Some NHS trusts develop their own clinical guidelines. However, the Bedside Clinical Guidelines have been produced and used for over twenty years in a significant number of NHS Trusts across the UK. The guidelines have been developed by the Bedside Clinical Guidelines Partnership (BCGP). They are currently created and edited as Microsoft Word documents, updated on an annual or biannual basis as new research is integrated into clinical practice.

Printing and distributing physical copies of the guidelines is costly, and the eBook versions are difficult to find on the internal intranet systems and hard to navigate (particularly on mobile devices). A more efficient and usable approach to delivering the Bedside Clinical Guidelines is required that utilises the flexibility of modern technology. Making the guidelines available in more accessible formats will also enable the BCGP to establish a broader user base and increase usage levels, especially with Junior Doctors and Nurse Practitioners.

\subsection{Previous work}

Studies to measure the impact and usability of computerised clinical guidelines implemented on mobile devices are limited. This is especially true for UK based studies that utilise a user-centred design approach. UK based studies are necessary to compare and contrast how these methods have worked within the UK healthcare system, which is mostly unique in its structure.

A study into mobile multimodal interaction and process-aware execution of clinical guidelines (Cossu et al. 2014) and two similar studies (Kwa et al. 2015, Payne et al. 2014) where a UCD process was implemented on a mobile device to present clinical guidelines to clinicians in a UK based hospital were identified. The studies provide evidence that a UCD approach utilised to develop an application used in a clinical setting can be successful and that a UCD approach works efficiently in a hospital setting. Even in pilot studies, applications that utilise a UCD approach allows clinicians to save time and access clinical guidelines in a user-friendly way. Unfortunately, the studies have limitations such as small sample sizes or focus primarily on a junior doctor cohort. Although they offer valid data for the implementation of a UCD mobile device application, it does require further study to establish how these applications could be used by all 
clinicians, across multiple disciplines. The authors conclude that the cost and perception of mobile device use in a hospital setting are barriers to adoption (Payne, Weeks and Dunning, 2014). The studies do produce a more usable system to access the hospital approved guidelines, but this improved usability is typically limited to the menu system of the application. Beyond the menu, users are often presented with the original guidelines in a Portable Document Format (PDF) or limited information, e.g. Specific dose levels of a drug. This creates numerous usability issues. Other relevant research, such as the production of the NASA GuideView system (lyengar et al. 2009) or research by Van der Velde on mobile access to Clinical guidelines (Velde et al. n.d.), highlights how clinical guidelines can be simplified and adapted to be more efficient, reduce cognitive load and be integrated into a usable system. Although this research highlights these factors, they were developed in a pre-smartphone era, which limits the transferability of some elements (such as the interface design and testing methods) into current research.

The studies offered an insight into how a UCD approach to developing mobile device applications for use in a hospital setting can be successful. This is especially true when considering the results, which show that these applications have made access to guidelines more efficient and usable. Attention must be given to displaying information in such a way as to relate to clinicians specifically. These studies also lead to further questions, such as how can a UCD approach make access to the guidelines more efficient, or even improve the efficiency of the guidelines themselves? How can a UCD approach work with all roles in a clinical environment? How can clinical guidelines information be structured and stored for efficient access and portability? There are further questions that should be considered, and that this is an emerging area of research that allows for numerous approaches to solving the problems discussed in this research.

\section{STUDY OUTLINE}

This research focusses on the intersection between human-computer interaction, clinical information delivery and mobile application development. Specifically, the study investigates how clinicians interact with information and utilise a user-centred design approach to examine methods of delivery on mobile devices. This project uses research in several related fields. This includes, but is not limited to, broader subjects such as Software Engineering; User-Centred Design; User Interface Design; Human Centred Design; Mobile Application Development and aspects of cognitive science, learning, and Human-Computer Interaction $(\mathrm{HCl})$.
The project also requires more specific areas of research; these include Medical Decision Support and associated technology; Technology acceptance in a hospital setting; Point of Care tools for Clinicians and Clinical Guideline research. Each stage of the study uses aspects from UCD methodology (Abras et al. 2004, usability.gov 2019), best practice design analysis and evaluation (Abras et al. 2004, Gerhardt-Powals 1996, Nielsen 1992, 1994a, b, usability.gov 2019), and software development methodologies (Fowler and Highsmith 2001). This included observations on clinical technology use, a survey to understand the technology and apps clinicians use, heuristic evaluations to ensure apps meet basic usability standards before testing; focus groups to gather feedback; System usability scales (SUS) (Brookes 1996) to measure any improvements in usability or any aspects that diminish usability.

This investigation focussed on the delivery of BCGs on mobile devices, via a user-centred design (UCD) approach (Abras et al. 2004) to inform the design of a BCG mobile app.

Observations were conducted at the Royal Stoke University Hospital to identify how or if clinical guidelines were being used. Clinicians across multiple departments were observed over three months between May and July 2018 (conducted inline with best practice (O'Reilly 2004, Potts 1993). The 'jotting note' method (Emerson et al. 1995) was adopted for observation recording with analysis focussing on identifying trends related to technology use and behaviour.

A survey was distributed to clinicians in three North West UK NHS Trusts $(n=\sim 1400)$ and medical students (3rd - 5th years) at Keele University $(n=\sim 300)$. The aim was to analyse technology use and identify design patterns and functionality in their preferred mobile apps. The survey collected data relating to the respondent's age; device ownership; role within the hospital (i.e. Consultant/Student); website use relevant to their role; app use pertinent to their position; time in the role and local guideline use. Specific App use (e.g. App Name) was collected via open-ended response.

To obtain functionality and design feedback for a prototype application from clinicians, Focus groups were conducted utilising both open discussion (Austin 1994, Gibbs 1997, Kitzinger 1995) and idea writing (VanGundy 1984). Sessions were time-sensitive (scheduling constraints inherent in clinical roles) and individual sessions, though preferred, were not possible. Open discussion sessions were audio-recorded and transcribed. The transcripts and outputs of the idea writing were then analysed using thematic analysis (Fereday and Muir-Cochrane 2006) 
Thirty-nine $\quad(n=39) \quad$ cognitive walkthrough sessions were conducted as a method of evaluation (Lewis and Wharton 1997) and further feedback. Participants were asked to find information within a prototype application by following clinical scenarios.

The Brookes System Usability Scale (SUS) (Brookes 1996) was also used to gather feedback in the form of a usability score. These sessions were audio-recorded and transcribed. The transcripts and outputs of the cognitive walkthrough sessions were then analysed using thematic analysis (Fereday and Muir-Cochrane 2006)

\section{RESULTS OVERVIEW}

\subsection{Observations}

Observing clinicians produced several interesting findings. These findings are discussed in previous work (Mitchell et al. 2020). A key theme of these findings was the necessity to present, as efficiently as possible, the information in a succinct manner. It is also necessary to ensure that clinicians are offered tools to prevent them from having to use multiple systems to complete simple tasks. An example of this would be finding prescribing information for a drug and then having to access another tool to calculate the dose for a specific patient.

\subsection{Survey}

The survey received one hundred and forty-six responses $(n=146)$. The survey results reported in previously published work (Mitchell et al. 2020) highlighted a large number of mobile applications in use by clinicians. Often clinicians used different tools to complete the same task (e.g. Digoxin drug dosage calculations). This highlights the lack of evidenced-based application development and the lack of NHS supported mobile applications.

\subsection{Focus Groups}

The focus groups, also reported in previously published work (Mitchell et al. 2020), highlighted the differing knowledge between experienced and junior clinicians. Another highlight was the various methods clinicians use for searching/filtering. The more experience clinicians seemed to use utilise acronyms inline with descriptions used in clinical practice. Examples of this include AF for Atrial Fibrillation or MI for Myocardial Infarction.

\subsection{Cognitive Walkthrough Sessions}

The cognitive walkthrough sessions are still being analysed. Early findings have proven that the application does not appear to introduce errors in clinical scenarios. Participants were able to quickly and efficiently access the information they required and apply it to further stages of the clinical scenarios. One key theme was the need to repeat information contained within clinical warnings with the content of the guideline. This enabled would enable users to see the warning information within context. However, this requires further analysis.

\subsection{System Usability Scale (SUS)}

At all stage of the study, the SUS score remained high (80+). The SUS score increased after each iteration, showing that users reacted positively to feedback informed changes.

\section{STUDY CONCLUSIONS}

Clinicians already use a wide range of applications, mainly on the iPhone, that are mostly unsupported and unchecked by the NHS. When designing for mobile, it is essential to design not only for the inherent strengths and weaknesses of the device but also for the context of use. Designing for in the moment use in a Hospital means designing for interruption and designing for users with specific expertise means including functionality that is counter-intuitive to standard design guidelines, e.g. using acronyms. Reflecting on the use of UCD itself in this domain, there are severe constraints related to limited access to clinicians and so traditional methods have required adaption. Future work, therefore, will consider the use of implicit feedback (usage logs) in order to gather feedback to inform user modelling and interface adaptation.

\section{ACKNOWLEDGEMENTS}

Thank you to Dr Ed de Quincey, Dr Charles Pantin, Dr Naveed Mustfa and Dr Sandra Woolley for their help and support. I would also like to thank all of the clinicians and students who took part in the various aspects of this study.

\section{REFERENCES}

Abras C, Maloney-Krichmar D and Preece J (2004) User-Centered Design.

Austin M (1994) Needs Assessment by Focus Groups. American Society for Training and Development.

Brookes J (1996) SUS: a "quick and dirty' usability Scale. .

Cossu F, Marrella A, Mecella M, Russo A, Kimani S, Bertazzoni G, Colabianchi A, Corona A, Luise AD, Grasso F and Suppa M (2014) Supporting Doctors through Mobile Multimodal Interaction and Process-Aware Execution of Clinical 
Guidelines. , 183 190. Available at: http://ieeexplore.ieee.org/document/6978608/.

Emerson RM, Fretz RI and Shaw LL (1995) Writing Ethnographic Fieldnotes.

Fereday $\mathrm{J}$ and Muir-Cochrane E (2006) Demonstrating Rigor Using Thematic Analysis: A Hybrid Approach of Inductive and Deductive Coding and Theme Development. International Journal of Qualitative Methods 5(1): 80-92.

Fowler M and Highsmith J (2001) The Agile Manifesto.

Gerhardt-Powals J (1996) Cognitive engineering principles for enhancing human-computer performance. International Journal of HumanComputer Interaction 8(2): 189-211.

Gibbs A (1997) What are focus groups? (19).

lyengar MS, Florez-Arango JF and Garcia CA (2009) GuideView: a system for developing structured, multimodal, multi-platform persuasive applications. 1.

Kitzinger J (1995) Qualitative Research: Introducing focus groups. BMJ 311(7000): 299302.

Kwa A, Carter M, Page D, Wilson T, Brown M and Baxendale B (2015) Nottingham University Hospital Guidelines App-Improving Accessiblity to 650 Hospital Clinical Guidelines. 220.

Lewis C and Wharton C (1997) Handbook of Human-Computer Interaction. Part IV: Evaluation of $\mathrm{HCl}$ (Software Practice and Experience2411994): 717-732.

Mitchell J, Quincey E de, Pantin C and Mustfa N (2020) The Development of a Point of Care
Clinical Guidelines Mobile Application Following a User-Centred Design Approach. 294-313.

Nielsen J (1992) Finding usability problems through heuristic evaluation. 373-380.

Nielsen J (1994a) Enhancing the explanatory power of usability heuristics. 152-158.

Nielsen J (1994b) Usability inspection methods. 413-414.

O'Reilly K (2004) Ethnographic Methods. 157-174.

Pantin C, Mucklow J, Rogers D, Cross M, Wall J and Partnership TBCG (2006) Bedside clinical guidelines: the missing link. Clinical Medicine 6(1): 98-104.

Payne KF, Weeks L and Dunning P (2014) A mixed methods pilot study to investigate the impact of a hospital-specific iPhone application (iTreat) within a British junior doctor cohort. Health informatics journal 20(1): 5973.

Potts C (1993) Software-engineering research revisited. IEEE Software 10(5): 19-28.

usability.gov (2019) User Centred Design, US Government. Available at: https://www.usability.gov/what-and-why/usercentered-design.html (accessed 20).

VanGundy AB (1984) Brain Writing for New Product Ideas: An Alternative To Brainstorming. Journal of Consumer Marketing 1(2): 67-74.

Velde ET van der, Atsma DE, Foeken $\mathrm{H}$, Witteman TA, Schalij MJ, 2003 and Wall EE van der (n.d.) Improvement in quality of care by mobile access to always-up-to-date clinical guidelines and documentation of the decision process. , 761 764. Available at: http://ieeexplore.ieee.org/document/1291267/. 